\title{
Construction of the Solution of the Caushy's Problem by the Riemann's Method for a Hyperbolic Equation
}

\author{
Akimov Andrey ${ }^{1}$, Kazakova Yevgeniya ${ }^{2}$, Vildyaeva Anzhelika ${ }^{3}$ \\ Department of Physics and Mathematics, Sterlitamak Branch of the Bashkir State University, Sterlitamak, Russia
}

Abstract. In this paper for the hyperbolic equation was constructed the four-parameter group and with the help of the group was found the solution of the Cauchy problem by the Riemann method for a hyperbolic equation.

Keywords: problem Cauchy, Riemann's function, hyperbolic equation, group analysis.

\section{INTRODUCTION}

Group analysis of differential equations is widely used in the study of equations of mathematical physics. This paper is of a synthetic nature, being a result of combining Riemann's method [1] for integrating second-order linear hyperbolic equations with Lie's classification [2] of such equations. One can find in [3] a detailed description of known methods of constructing Riemann's function (called in [3] the Riemann-Green function) for particular types of equations. Specifically, six methods are described there. For example, one method has been proposed Hadamard. He showed that the function of the Riemann coincides with a coefficient of the logarithmic term in the elementary solution of the equation [4]. The group-theoretical approach presented below provides the seventh method. Using the results for the group classification of homogeneous hyperbolic equation of the second order, it was suggested to find a function of Riemann using the symmetries of the equation.

\section{Preliminaries}

Let's consider the following hyperbolic equation of the second order:

$L u=x^{3} u_{x x}-y^{2} x u_{y y}-4 y^{3} u=0$

in an open domain $D$, which is bounded by curves of $A C(y=2 x), C B(y x=1)$ and with the section $A B(y=1)$.

Let's pose the problem of Cauchy: Find in the domain $D$ function $u(x, y)$, satisfying the conditions

$$
\begin{aligned}
& u(x, y) \in C(\bar{D}) \cap C^{1}(D \cup A B) \cup C^{2}(D) ; \\
& L u(x, y) \equiv 0, \quad(x, y) \in D ; \\
& \left.u\right|_{y=1}=\varphi(x),\left.\frac{\partial u}{\partial y}\right|_{y=1}=\psi(x), \frac{1}{2} \leq x \leq 1
\end{aligned}
$$

where $\varphi(x), \psi(x)$ - given sufficiently smooth functions.

With the help of the change of variables $\xi=x y, \eta=\frac{y}{x}$ equation (1) leads to the canonical form:

$u_{\xi \eta}-\frac{1}{2 \xi} u_{\eta}+\eta u=0$

\footnotetext{
${ }^{1}$ Corresponding Author: Andakm@ rambler.ru
} 
American Research Journal of Mathematics, Volume 1, Issue 2, April 2015

ISSN 2378-704X

To solve the problem we use the method of Riemann, which is based on the following identity:

$$
2\left(v L u-u L^{*} v\right)=\left(v u_{\eta}-u v_{\eta}+2 a u v\right)_{\xi}+\left(v u_{\xi}-u v_{\xi}+2 b u v\right)_{\eta}
$$

where

$$
\begin{aligned}
& L u=u_{\xi \eta}+a(\xi, \eta) u_{\xi}+b(\xi, \eta) u_{\eta}+c(\xi, \eta) u=f(\xi, \eta), \\
& L u=u_{\xi \eta}+a(\xi, \eta) u_{\xi}+b(\xi, \eta) u_{\eta}+c(\xi, \eta) u=f(\xi, \eta)
\end{aligned}
$$

$L^{*} v=v_{\xi \eta}-(a v)_{\xi}-(b v)_{\eta}+c v-$ adjoint with $L u$ differential operator; $G$ - domain of integration with piecewise-smooth contour $\Gamma$.

Integrating both sides of (6) in the domain of $G$ and, using the formula of Ostrogradskii, obtain

$$
2 \iint_{G}\left(v L u-u L^{*} v\right) d \xi d \eta=\int_{\Gamma}\left[-\left(v u_{\xi}-u v_{\xi}+2 b u v\right) d \xi+\left(v u_{\eta}-u v_{\eta}+2 a u v\right) d \eta\right] .
$$

Riemann's method reduces the problem of integrating the equation (1) to construct an auxiliary Riemann's function $v=R\left(\xi, \eta ; \xi_{0}, \eta_{0}\right)$, that satisfies the homogeneous adjoint equation (the variables $\xi, \eta$ ):

$$
L^{*} R=0
$$

and the following conditions on the characteristics of:

$$
\begin{aligned}
& \left.\left(R_{\eta}-a R\right)\right|_{\xi=\xi_{0}}=0, \\
& \left.\left(R_{\xi}-b R\right)\right|_{\eta=\eta_{0}}=0, \\
& R\left(\xi_{0}, \eta_{0} ; \xi_{0}, \eta_{0}\right)=1 .
\end{aligned}
$$

The Riemann's formula in general is for the solution of equation (7) has the form

$$
\begin{aligned}
& u\left(\xi_{0}, \eta_{0}\right)=\frac{(u R)_{P}+(u R)_{Q}}{2}-\frac{1}{2} \int_{P Q}-\left(R u_{\xi}-u R_{\xi}+2 b u R\right) d \xi+\left(R u_{\eta}-u R_{\eta}+2 a u R\right) d \eta+ \\
& +\iint_{G} R f d \xi d \eta
\end{aligned}
$$

where the double integral is taken over the domain bounded by the characteristics $\xi=\xi_{0}, \eta=\eta_{0}$ and the curve $\gamma(\mathrm{PQ})$. The solution of the Goursat problem (8) is unique.

\section{MAin Results}

In our case, the equation adjoint equation (5) has the form

$u_{\xi \eta}+\frac{1}{2 \xi} u_{\eta}+\eta u=0$

the function of Riemann $v=R\left(\xi, \eta ; \xi_{0}, \eta_{0}\right)$. Let's note that in our case the desired function of Riemann satisfies the following conditions on the characteristics: 


$$
\left.R\right|_{\eta=\eta_{0}}=\sqrt{\frac{\xi_{0}}{\xi}},\left.R\right|_{\xi=\xi_{0}}=1, \quad R\left(\xi_{0}, \eta_{0} ; \xi_{0}, \eta_{0}\right)=1
$$

The symmetry operator of the homogeneous equation (10) has the form [4]:

$$
X=v(\xi) \frac{\partial}{\partial \xi}+w(\eta) \frac{\partial}{\partial \eta}+\tau(\xi, \eta) u \frac{\partial}{\partial u} .
$$

Thus, as follows from [5], must be done the following relations:

$$
\begin{gathered}
\frac{\partial \tau}{\partial \xi}+\frac{\partial(b v)}{\partial \xi}+w \frac{\partial b}{\partial \eta}=0, \quad \frac{\partial \tau}{\partial \eta}+\frac{\partial(a w)}{\partial \eta}+v \frac{\partial a}{\partial \xi}=0 \\
\frac{\partial^{2} \tau}{\partial \xi \partial \eta}+a \frac{\partial \tau}{\partial \xi}+b \frac{\partial \tau}{\partial \eta}+\frac{\partial(c v)}{\partial \xi}+\frac{\partial(c w)}{\partial \eta}=0
\end{gathered}
$$

Substituting in this case $a=0, b=-\frac{1}{2 \xi}, c=\eta$, we'll obtain the following relations

$$
v=C_{2} \xi+C_{4}, w=C_{2} \frac{\eta}{2}+C_{3} \frac{1}{\eta}, \tau=C_{1}-\frac{C_{2}}{2},
$$

where $C_{1}, C_{2}, C_{3}, C_{4}$ - arbitrary constants. Thus, equation (5) in this case allows operators four parameter group:

$$
X_{1}=\frac{\partial}{\partial \xi}, X_{2}=\frac{1}{\eta} \frac{\partial}{\partial \eta}, X_{3}=\xi \frac{\partial}{\partial \xi}+\frac{\eta}{2} \frac{\partial}{\partial \eta}-\frac{1}{2} u \frac{\partial}{\partial u}, X_{4}=u \frac{\partial}{\partial u}
$$

Let's construct a linear combination of these operators

$$
X=\alpha_{1} X_{1}+\alpha_{2} X_{2}+\alpha_{3} X_{3}+\alpha_{4} X_{4}
$$

where $\alpha_{1}, \alpha_{2}, \alpha_{3}, \alpha_{4}-$ arbitrary constants.

Following [6], we require invariance characteristics $\xi=\xi_{0}$ and $\eta=\eta_{0}$ regarding construction of the operators:

$$
X\left(\xi-\xi_{0}\right)=0, X\left(\eta-\eta_{0}\right)=0 \text {. }
$$

If we choose $\alpha_{3}=1$, we'll get $\alpha_{2}=-\frac{\eta_{0}^{2}}{2}, \alpha_{2}=-\xi_{0}$. Then the resulting operator takes the form

$$
X=\left(\xi-\xi_{0}\right) \frac{\partial}{\partial \xi}+\left(\frac{\eta}{2}-\frac{\eta_{0}^{2}}{2 \eta}\right) \frac{\partial}{\partial \eta}+u \frac{\partial}{\partial u}
$$

Invariants of this operator have the form $I_{1}=\left(\xi-\xi_{0}\right)\left(\eta^{2}-\eta_{0}^{2}\right), I_{2}=\frac{u}{\xi-\xi_{0}}$, therefore we'll seek the solution of equation (5) as a function of $R=f(z) g(r)$, where $z=\left(\xi-\xi_{0}\right)\left(\eta^{2}-\eta_{0}^{2}\right), r=\xi-\xi_{0}$. As a result of substitution of $R$ in equation (10), it splits into two ordinary differential equations 
American Research Journal of Mathematics, Volume 1, Issue 2, April 2015

ISSN 2378-704X

$$
\begin{gathered}
2 z f^{\prime \prime}(z)+2 f^{\prime}(z)+f(z)=0, \\
2\left(r+\xi_{0}\right) g^{\prime}(r)+g(r)=0 .
\end{gathered}
$$

The solutions of the obtained equations are functions

$$
f=J_{0}\left(\sqrt{2\left(\xi-\xi_{0}\right)\left(\eta^{2}-\eta_{0}^{2}\right)}\right), g=\frac{1}{C \sqrt{\xi}},
$$

where $J_{0}(\cdot)$ - Besel's function of the first kind of order zero, $C-$ an arbitrary constant.

Then satisfied with the decision $R=f(z) g(r)$ of the conditions (6), we obtain the Riemann's function

$$
\left.R\left(\xi, \eta, \xi_{0}, \eta_{0}\right)=\sqrt{\frac{\xi_{0}}{\xi}} J_{0}\left(\sqrt{2\left(\xi-\xi_{0}\right)\left(\eta^{2}-\eta_{0}^{2}\right.}\right)\right) .
$$

Since $x=\sqrt{\frac{\xi}{\eta}}, y=\sqrt{\xi \eta}$, then

$\left.\frac{\partial u}{\partial \xi}\right|_{\xi \eta=1}=\frac{1}{2} \frac{\partial u}{\partial x}+\left.\frac{1}{2 \xi} \frac{\partial u}{\partial y}\right|_{\xi \eta=1},\left.\frac{\partial u}{\partial \eta}\right|_{\xi \eta=1}=-\frac{\xi^{2}}{2} \frac{\partial u}{\partial x}+\left.\frac{\xi}{2} \frac{\partial u}{\partial y}\right|_{\xi \eta=1}$

$\left.\frac{\partial u}{\partial \xi}\right|_{\xi \eta=1}=\frac{1}{2} \varphi^{\prime}(\xi)+\frac{1}{2 \xi} \psi(\xi),\left.\frac{\partial u}{\partial \eta}\right|_{\xi \eta=1}=-\frac{\xi^{2}}{2} \varphi^{\prime}(\xi)+\frac{\xi}{2} \psi(\xi)$

and

$\left.u\right|_{\xi \eta=1}=\varphi(\xi)$

Substituting in the formula (9) $a=0, b=-\frac{1}{2 \xi}, f=0$ and, taking into account, that

$u(P)=\varphi\left(\xi_{0}\right), u(Q)=\varphi\left(\frac{1}{\eta_{0}}\right), R(P)=R\left(\xi_{0}, \frac{1}{\xi_{0}} ; \xi_{0}, \eta_{0}\right)=1, R(Q)=R\left(\frac{1}{\eta_{0}}, \eta_{0} ; \xi_{0}, \eta_{0}\right)=\sqrt{\xi_{0} \eta_{0}}$,

we'll get

$$
\begin{gathered}
u\left(\xi_{0}, \eta_{0}\right)=\frac{\varphi\left(\xi_{0}\right)}{2}+\frac{\sqrt{\xi_{0} \eta_{0}}}{2} \varphi\left(\frac{1}{\eta_{0}}\right)+\frac{\sqrt{\xi_{0}}}{4} \int_{\xi_{0}}^{\frac{1}{\eta_{0}}} \frac{\varphi(\xi)}{\sqrt{\xi^{3}}} J_{0}\left(\left(\xi-\xi_{0}\right)\left(\frac{1}{\xi^{2}}-\eta_{0}^{2}\right)\right) d \xi- \\
-\frac{\sqrt{\xi_{0}}}{2} \int_{\xi_{0}}^{\frac{1}{\eta_{0}}} \frac{\psi(\xi)}{\sqrt{\xi^{3}}} J_{0}\left(\left(\xi-\xi_{0}\right)\left(\frac{1}{\xi^{2}}-\eta_{0}^{2}\right)\right) d \xi-
\end{gathered}
$$




$$
-\frac{\sqrt{\xi_{0}}}{2} \int_{\xi_{0}}^{\frac{1}{\eta_{0}}} \frac{\psi(\xi)}{\sqrt{\xi^{7}}}\left(3 \xi-\xi^{3} \eta_{0}^{2}-2 \xi_{0}\right) J_{1}\left(\left(\xi-\xi_{0}\right)\left(\frac{1}{\xi^{2}}-\eta_{0}^{2}\right)\right) d \xi
$$

Returning to the old variables $x$ and $y$, we'll get the solution of the Cauchy's problem

$$
\begin{gathered}
u(x, y)=\frac{\varphi(x y)}{2}+\frac{y}{2} \varphi\left(\frac{x}{y}\right)+\frac{\sqrt{x y}}{4} \int_{x y}^{\frac{x}{y}} \frac{\varphi(z)}{\sqrt{z^{3}}} J_{0}\left((z-x y)\left(\frac{1}{z^{2}}-\left(\frac{y}{x}\right)^{2}\right)\right) d z- \\
-\frac{\sqrt{x y}}{2} \int_{x y}^{\frac{x}{y}} \frac{\psi(z)}{\sqrt{z^{3}}} J_{0}\left((z-x y)\left(\frac{1}{z^{2}}-\left(\frac{y}{x}\right)^{2}\right)\right) d z- \\
-\frac{\sqrt{y}}{2 x} \int_{x y}^{\frac{x}{y}} \frac{\varphi(z)}{z^{3}}\left(\frac{3 z x^{2}-z^{3} y^{2}-2 x^{3} y}{\sqrt{2(z-x y)(x-z y)}}\right) J_{1}\left((z-x y)\left(\frac{1}{z^{2}}-\left(\frac{y}{x}\right)^{2}\right)\right) d z
\end{gathered}
$$

Theorem. If the functions $\varphi(x) \in C^{2}\left[\frac{1}{2} ; 1\right] \psi(x) \in C^{2}\left[\frac{1}{2} ; 1\right]$, then the Cauchy's problem for equation (1) has a unique solution, which is defined by (12).

\section{REFERENCES}

[1] G. F. B. Riemann. Uber die Fortpflanzung ebener Luftwellen von endlicher Schwingungsweite. Abhandl. Konigl. Ges. Wiss. Gottingen, 8:43-65, 1860.

[2] S. Lie. Uber die Integration durch bestimmte Integrale von einer Klasse linearer partieller Differentialgleichungen. Archiv for Matematik og Naturvidenskab (Abbr. Arch. for Math.), 6, Heft 3:328-368, 1881. English transl., CRC Handbook of Lie Group Analysis of Differential Equations, Vol. 2: Applications in Engineering and Physical Sciences, ed. N.H. Ibragimov, CRC Press, Boca Roton, 1995. Reprinted also in the book Lie group analysis: Classical heritage, ed. N.H. Ibragimov, ALGA Publications, Karlskrona, 2004.

[3] E. T. Copson. On the Riemann-Green function. Arch. Rational Mech. Anal., 1:324-348, 1958.

[4] J. Hadamard, "Lectures on Cauchy's problem in linear partial differential equations", Dover, reprint (1952) (Translated from French

[5] L. V. Ovsyannikov. Group properties of differential equations. Siberian Branch, USSR Academy of Sciences, Novosibirsk, 1962. (Russian).

[6] N. H. Ibragimov. Group analysis of ordinary differential equations and the invariance principle in mathematical physics (for the $150^{\text {th }}$ anniversary of Sophus Lie). Uspekhi Mat. Nauk, 47, No. 4:83-144, 1992. English transl., Russian Math. Surveys, 47:2 (1992), 89-156.

[7] P. J. Olver. Applications of Lie groups to differential equations. Springer-Verlag, New York, 1986. 2nd ed., 1993. 